\title{
pH-Sensitive Hybrid Nanoparticles for the Controlled Release of Tyrosine Kinase Inhibitors
}

\author{
$\underline{\text { Andra-Sorina Tatar }}{ }^{1}$, Simion Astilean', Sanda Boca ${ }^{1, *}$ \\ ${ }^{1}$ Nanobiophotonics and Laser Microspectroscopy Center, Interdisciplinary Research Institute on Bio-Nano-Sciences, \\ Babes-Bolyai University, 42 Treboniu Laurian street, Cluj-Napoca, Romania; \\ andra.tatar@ubbcluj.ro, simion.astilean@phys.ubbcluj.ro, *sanda.boca@phys.ubbcluj.ro
}

\section{Extended Abstract}

Smart nanomaterials are emerging as a promising tool for use in biomedical applications, due to their special properties that render them easily controllable and reactive to relevant stimuli. As nanomedicine is gaining more focus in medical research, the development of such smart, stimuli-sensitive nanoparticles with controllable behaviour is a logical development step which brings certain additional advantages above those already possessed. Thus, by ensuring that the cargo of the nanoparticles (be it of therapeutic nature or an investigative dye) is delivered and released at the target with a precision that can be focused up to the level of cell organelles, the side effects can be considerably averted, the necessary dose heavily reduced, and the costs lowered.

A main object of focus in the field of smart nanomaterials is a class of so-called 'smart polymers', organic molecules with remarkable physical-chemical properties, namely reactivity to certain stimuli such as variations in temperature, acidity, salt concentrations, or redox potential [1], leading to changes in solubility, size, structural integrity, and so on. Additionally, by their association with inorganic nanoparticles such as magnetic or plasmonic nanoparticles, the range of unique properties is further enriched and the possibilities become endless [2].

Herein, we focus on the development of hybrid gold-core - $\mathrm{pH}$-sensitive-corona nanoparticles that can serve for the controlled release of Lapatinib, a Tyrosine Kinase Inhibitor (TKI) drug that acts at the level of the Epidermal Growth Factor Receptor (EGFR), a transmembrane protein involved in cell adhesion and proliferation and is responsible for multiple types of cancer such as epithelial, pulmonary or breast cancer [3]. In this regard, gold nanoparticles of spherical shape and $60 \mathrm{~nm}$ average sizes were loaded with the drug and conjugated with various stimuli-responsive polymers such as Pluronic, poly lactic-co-glycolic acid (PLGA) and poly-histidine (pHis). Physical-chemical characterization of the particles, assessment of the drug loading efficiency and subsequent stimulitriggered controlled release in conditions mimicking the lyzosomal microenvironment were performed by UV-VisNIR extinction spectroscopy and Dynamic Light Scattering (DLS). Our results illustrate that the chemical stability and drug releasing capacity of the proposed nanocarrier systems depend on the polymeric coating physical-chemical properties. The obtained results might represent a step forward in the development of promising agents for the efficient delivery and release of TKIs, which would have a significant therapeutic potential and less side effects than the non-conjugated drugs.

Acknowledgement: This work was supported by project GTC 31369/2020 from the Babes-Bolyai University.

\section{References}

[1] M. Mackiewicz, J. Romanski, K. Drabczyk, E. Waleka, Z. Stojek, and M. Karbarz, "Degradable, thermo-, pH- and redox-sensitive hydrogel microcapsules for burst and sustained release of drugs," Int. J. Pharm., vol. 569, p. 118589, Oct. 2019, doi: 10.1016/j.ijpharm.2019.118589.

[2] G. Guan, M. Wu, and M. Han, "Stimuli-Responsive Hybridized Nanostructures," Adv. Funct. Mater., vol. 30, no. 2, p. 1903439, Jan. 2020, doi: 10.1002/adfm.201903439.

[3] E. Kovacs, J. A. Zorn, Y. Huang, T. Barros, and J. Kuriyan, "A Structural Perspective on the Regulation of the Epidermal Growth Factor Receptor," Annu. Rev. Biochem., vol. 84, no. 1, pp. 739-764, Jun. 2015, doi: 10.1146/annurev-biochem-060614-034402. 